\title{
First evidence of the finite horizontal extent of the optical turbulence layers. Implications for new adaptive optics techniques
}

\author{
E. Masciadri ${ }^{1}$, R. Avila ${ }^{2}$, and L. J. Sánchez ${ }^{1}$ \\ 1 Instituto de Astronomía, Universidad Nacional Autónoma de México, Apartado Postal 70-264, \\ 04510, D.F. México \\ 2 Instituto de Astronomía (Campus Morelia), Universidad Nacional Autónoma de México, Apartado Postal 72-3, \\ 58089 Morelia Michoacan, México
}

Received 29 May 2001 / Accepted 7 November 2001

\begin{abstract}
It was recently shown (Masciadri et al. 2000; Masciadri 2001a), using a numerical simulation (Meso-Nh model), that considering horizontally uniform $C_{\mathrm{N}}^{2}$ can have severe consequences. For example, the integration of the $C_{\mathrm{N}}^{2}$ along lines of sight different from the zenith gives variations that can be larger than $0.50^{\prime \prime}$. In this paper we try to validate this result following two approaches. (a) numerical approach: we compare measured and simulated $C_{\mathrm{N}}^{2}$ profiles. The first ones were obtained with a Generalized Scidar (GS) and the latter are simulated with the Meso-Nh model at the same azimuth and elevation as those of the observations. Further measurements (DIMM, mast and balloons) are considered in order to support the comparison. (b) experimental approach: we compare $C_{\mathrm{N}}^{2}$ profiles measured (almost simultaneously) by a GS along different lines of sight. The results of this study show that (1) the horizontal size of the turbulent layers can be finite, (2) the simulations and the measurements are well correlated and (3) for the first time, we show that the model can reproduce observed seeing values that vary as much as $0.50^{\prime \prime}$ during the same night. This definitely shows that the numerical simulations are a useful tool in the context of the turbulence characterization for astronomical applications. Finally, we discuss the implications that a finite horizontal size of the turbulent layers could have on new adaptive optics techniques, particularly in applications to the extremely large size telescopes.
\end{abstract}

Key words. atmospheric effects - methods: numerical - site testing - techniques: interferometric

\section{Introduction}

It is well known (Dalaudier et al. 1994; Marks et al. 1996; Marks et al. 1999) that the optical turbulence $\left(C_{\mathrm{N}}^{2}\right)$ is concentrated, with respect to the vertical direction, in thin layers placed at different altitudes in the troposphere (up to $\sim 20 \mathrm{~km}$ ) and having a vertical size of some tens of meters. Such a structure is resolved by instruments such as those carried aboard the balloons that have a resolution of some meters. Optical instruments such as the Generalized Scidar (GS) cannot resolve such thin layers because their typical resolution is of about $1 \mathrm{~km}$. This fact has to be taken into account when the measurements provided by the two instruments are compared. It is usual to make a convolution of the balloon $C_{\mathrm{N}}^{2}$ profiles so as to obtain a lower resolution similar to that of the Scidar. In this way, spatial turbulent distributions having equivalent characteristics can be compared.

Send offprint requests to: E. Masciadri, e-mail: elena@astroscu.unam.mx
This means that we have to distinguish between the real structure of the turbulence and the structure resolved by different instruments (an important point that is frequently omitted). In the context of the adaptive optics applications it is extremely important to define the typical spatio-temporal characteristics of such layers. Many studies were done on the impact of the vertical structure of the turbulence on high angular resolution techniques. The multi-conjugated adaptive optics (MCAO), for example, is based on the hypothesis that the turbulence energy is localized in a few layers (Beckers 1988; Tallon \& Foy 1990; Tallon et al. 1992). This technique is based on the idea that a more efficient wavefront correction can be obtained by conjugating a small number of deformable mirrors (DM) to a few turbulent layers in the atmosphere. The principal advantage is that the residual isoplanatic angle obtained after wavefront correction is larger than that obtained with a classical procedure (Racine \& Ellerbroek 1995). The Laser Guide Star (LGS) technique, proposed to attain a large sky coverage 


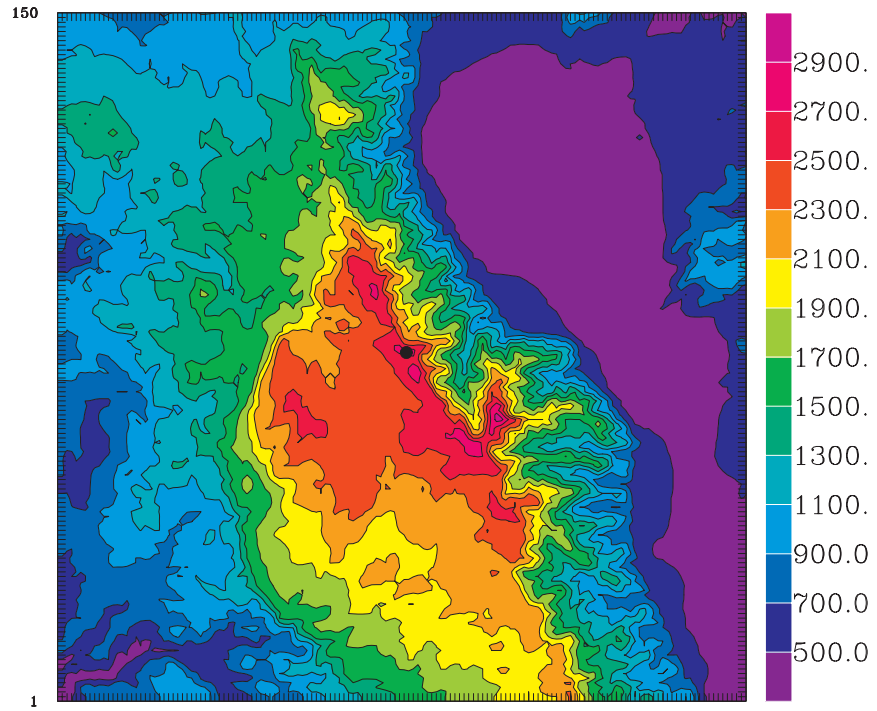

Fig. 1. Orographic model of San Pedro Mártir region. A domain of $60 \mathrm{~km} \times 60 \mathrm{~km}$ corresponding to $150 \times 150$ grid points is shown. The horizontal resolution is $400 \mathrm{~m}$. The black point represents the San Pedro Mártir site. The color table shows the graduation in altitude.

for the correction of the wavefront perturbations, depends strongly on the number of turbulent layers (Tallon \& Foy 1990). More precisely (Ragazzoni et al. 1999b), the number of LGS cannot be lower than the number of turbulent layers (or the DMs necessary for a full wavefront correction) to invert the system of equations that describe the $3 \mathrm{D}$ maps of the atmospherical perturbations. We precise here that the residual turbulence located far away from the DMs can have a non-negligable effect on the correction of the wavefront. Curiously, not much is known about the typical horizontal size of the turbulent layers and the implications that this characteristic could have on adaptive optics techniques. In previous studies (Masciadri et al. 2000; Masciadri 2001a) it was shown, using a numerical simulation (Meso-Nh model), that the integration of the $C_{\mathrm{N}}^{2}$ profile along lines of sight different from the zenith gives seeing differences that can be larger than $0.50^{\prime \prime}$. This is a sizable quantity that suggests the need for a deeper investigation.

In this paper (Sect. 2) we intend to attack this problem following two approaches. (1) We compare measured and simulated $C_{\mathrm{N}}^{2}$ profiles along the same lines of sight. The measured profiles are provided by a Generalized Scidar (Fuchs et al. 1998; Avila et al. 1997; Avila et al. 1998) at the focus of a $2.1 \mathrm{~m}$ telescope during a site testing campaign on 16-21 May 2000 in the Observatorio Astronómico Nacional de San Pedro Mártir (Mexico) $(31.0441 \mathrm{~N}, 115.4569 \mathrm{~W})$ and the simulations are provided by the Meso-Nh model (Masciadri et al. 1999a; Masciadri et al. 1999b). (2) We compare $C_{\mathrm{N}}^{2}$ profiles retrieved with the GS from the scintillation of different binary stars observed during the same night (21/22 May 2000) at different lines of sight. To support the comparison between measurements and simulations, further integrated and local measurements provided by DIMM (Differential Image Motion Monitor) balloons launched near by the Observatory and a mast of $16 \mathrm{~m}$ are used.

In order to give a reliable comparison between measurements and simulations we modified the code so as to give averaged estimations of the optical turbulence calculated over simulations of some hours. We recall that the previous results (Masciadri et al. 2000; Masciadri 2001a) were not the result of an average. They were retrieved by outputs obtained at an instant $t^{*}$ during the simulation (usually $t^{*}$ was at about 3 hours after the start of the simulation). Finally, for the first time, we show that the Meso-Nh model reproduces different seeing values (varying as much as $0.50^{\prime \prime}$ ) observed during the same night. In Sect. 3 we discuss the potential implications that the results of this study could have on adaptive optics techniques and in Sect. 4 we present the conclusions of this study.

\section{Horizontal spatial variability of the optical turbulence $\mathrm{C}_{\mathrm{N}}^{2}$}

During the site testing campaign in San Pedro Mártir Observatory, measurements expressly planned for the validation of the Meso-Nh model were done. $C_{\mathrm{N}}^{2}$ profiles were provided by a Generalized Scidar above the astronomical site and, almost simultaneously, meteorological balloons adapted to the measurement of classical meteorological parameters $(T, p, V)$ were launched about $80 \mathrm{~km}$ from the Observatory in an upstream position with respect to the principal wind direction. The place (Pta. Colonet) where the balloons were launched was chosen near the coast (to the west of the Observatory) so that the soundings sample the whole vertical atmosphere $[0-20] \mathrm{km}$. These last measurements are used to initialize the Meso-Nh model. More details on the site testing campaign will be presented in a forthcoming paper. Figure 1 shows the orographic model over which we simulate the optical turbulence. The computational region extends over a surface of $60 \mathrm{~km} \times 60 \mathrm{~km}$ and the horizontal resolution of the model is of $400 \mathrm{~m}$.

\subsection{Numerical validation}

The measurements obtained during the night of 16-17 May are analyzed. Figure 2 (on the left) shows the temporal evolution of the $C_{\mathrm{N}}^{2}$ profiles measured by the GS during this night. Figure 2 (on the right) shows the seeing measured by a DIMM and a 16-m-high mast placed at about $600 \mathrm{~m}$ from the $2.1 \mathrm{~m}$ telescope. The seeing retrieved from the mast is calculated in the $[2-16] \mathrm{m}$ interval (Sánchez et al. 2000). We select two time intervals in which we have both measurements of $C_{\mathrm{N}}^{2}$ profiles provided by the GS and seeing estimations provided by a DIMM. In the time interval [7/8] UT the DIMM measures an averaged seeing of about $1.70^{\prime \prime}$ and in the time interval [11/12] UT a seeing of about $0.91^{\prime \prime}$. In the following we will call [7/8] interval $A$ and $[11 / 12]$ interval $B$. At 06:58 UT a balloon was launched from the Observatory. This balloon 
(A)

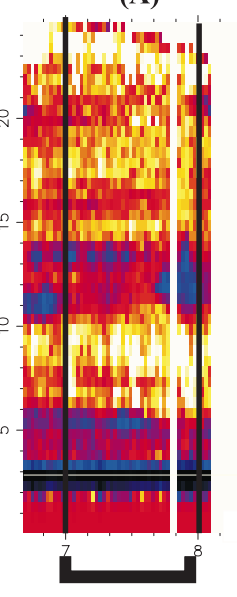

(B)

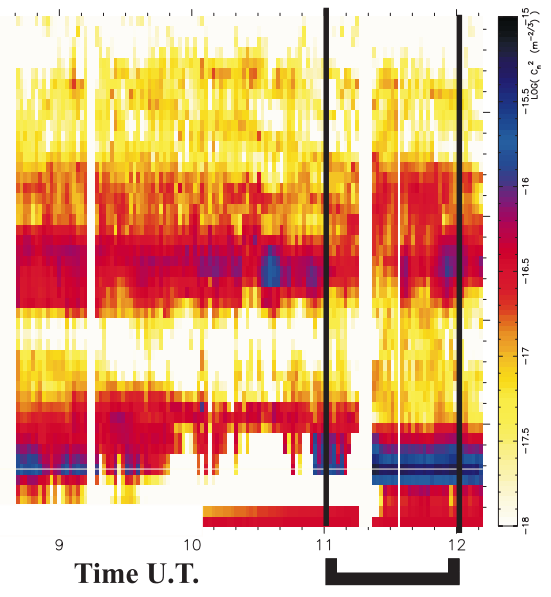

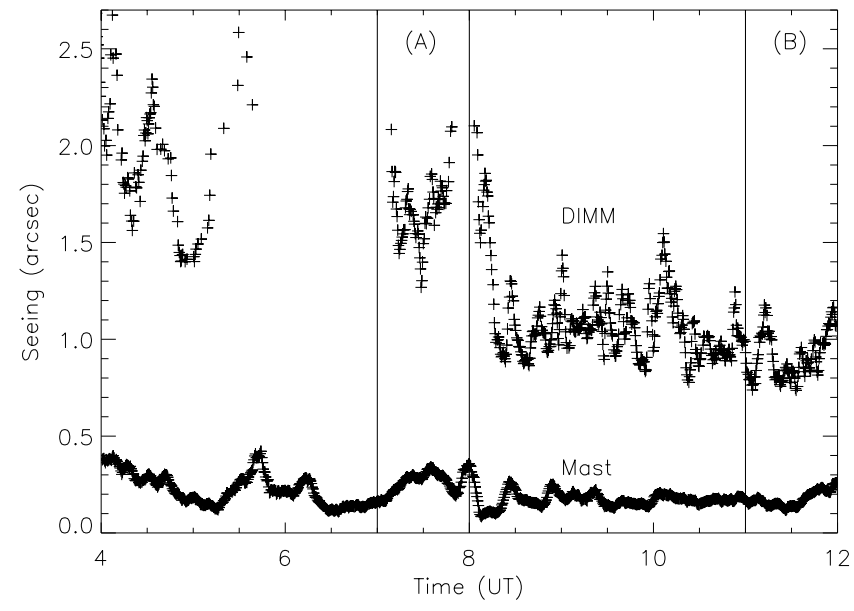

Fig. 2. On the left: temporal evolution of the $C_{\mathrm{N}}^{2}$ profiles measured by the GS during the night 16/17 May 2000. The intervals $A$ and $B$ are marked with vertical lines. On the right: seeing measured by a DIMM (upper line) and by a 16-m-high mast (bottom line). The seeing measured by the mast is calculated in the interval $[2-16] \mathrm{m}$.

measured the classical meteorological parameters and was adapted to measure the $C_{\mathrm{N}}^{2}$ too. As in the case of the GS, we compare the $C_{\mathrm{N}}^{2}$ profile measured by this balloon with the simulations.

We initialize the Meso-Nh model with the radiosoundings launched from Pta. Colonet at 05:09 UT to simulate the $C_{\mathrm{N}}^{2}$ in the interval $A$ and with the analysis of the European Centre for Medium Weather Forecasting (ECMWF) calculated at 12:00 UT in the coordinates $(31.5 \mathrm{~N}, 118.0 \mathrm{~W})$ to simulate the $C_{\mathrm{N}}^{2}$ in the interval $B$. The availability of the initial conditions for the Meso-Nh model are a further criterion in the selection of the intervals $A$ and $B$. The binary star observed during the interval $A$ is $\zeta$ UMa $\left(\alpha_{2000}=13^{\mathrm{h}} 23^{\prime} 55^{\prime \prime}, \delta_{2000}=54^{\circ} 55^{\prime} 14^{\prime \prime}\right)$ and the binary observed during the interval $B$ is $95 \mathrm{Her}$ $\left(\alpha_{2000}=18^{\mathrm{h}} 1^{\prime} 31^{\prime \prime}, \delta_{2000}=21^{\circ} 35^{\prime} 43^{\prime \prime}\right)$.

The polar sky map of Fig. 3 shows the path in the sky of the two binary stars ( $\zeta \mathrm{UMa}$ and 95Her) during the observation of the night $16 / 17$ with respect to the azimuth (0 degrees at the North, 90 degrees at the East) and to the zenith distance $\theta$. The centre of the polar sky maps is the zenith. Each circle selects the points above the same angle $\theta$. The circle with the maximum radius is the horizon. The black points mark the position of the stars during the interval $A$ and $B$ (see Fig. 2 on the left). During the interval $A$, the GS was scanning the atmosphere at about $\theta=32$ degrees, and during the interval $B$ at about $\theta=10$ degrees.

Figure 4, on the left, shows the $C_{\mathrm{N}}^{2}$ profiles measured by the GS (bold line) and simulated by the Meso-Nh model (thin line) during the interval $A$. The $C_{\mathrm{N}}^{2}$ profile provided by the balloon launched from the Observatory is shown with the dotted line. Figure 4, on the right, shows the $C_{\mathrm{N}}^{2}$ measured by the GS (bold line) and simulated by Meso-Nh (thin line) during the interval $B$. The simulation outputs are averaged over 3 simulation hours. Table 1 lists the values of the seeing calculated in the intervals $A$ and $B$ in

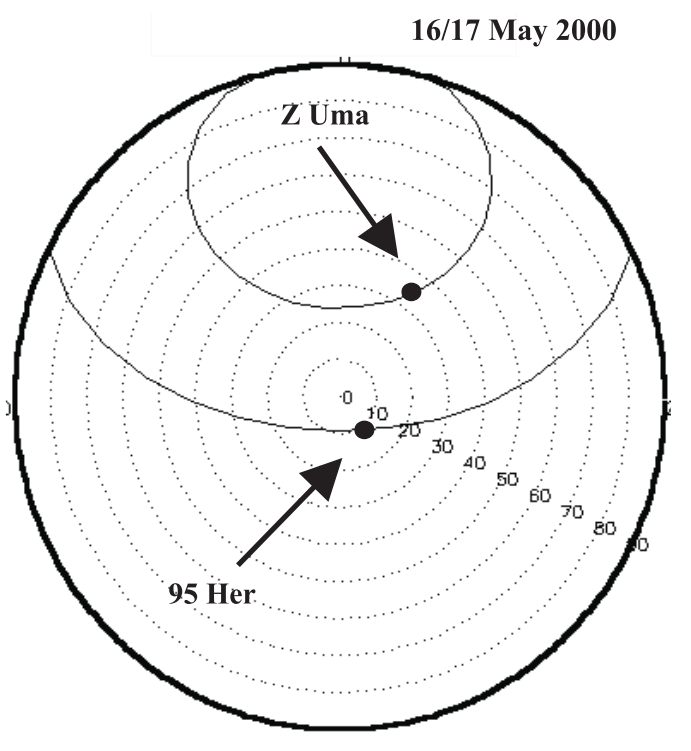

Fig. 3. Polar sky maps representing the path in the sky of the binary stars observed during the night 16/17 in the intervals $A$ and $B$ (see text). The centre of the polar maps is the zenith. Each circle (increment of 10 degrees) selects the points above the same angle $\theta$. The largest circle is the horizon.

the first $10 \mathrm{~km}\left(\varepsilon_{[0-10]}\right)$, between $10 \mathrm{~km}$ and the top of the atmosphere $(\varepsilon[10-20])$ and over the whole atmosphere $(\varepsilon$ тот $)$. In the Cols. 5-7 are shown the values of the seeing estimated by the DIMM, the mast and the subtraction (DIMM - mast). This last value is calculated linearly with respect to the $C_{\mathrm{N}}^{2}$. The last four columns show the difference of the seeing calculated between the Meso-Nh model and the GS (MG), the Meso-Nh model and the DIMM (MD), the GS and the DIMM (GD) and the GS and the balloons (GB). We note that the balloon was launched from Vallecitos (at an altitude of $2400 \mathrm{~m}$ ), a tree-clear area close to the Observatory at about $3 \mathrm{~km}$ from the $2.1 \mathrm{~m}$ 

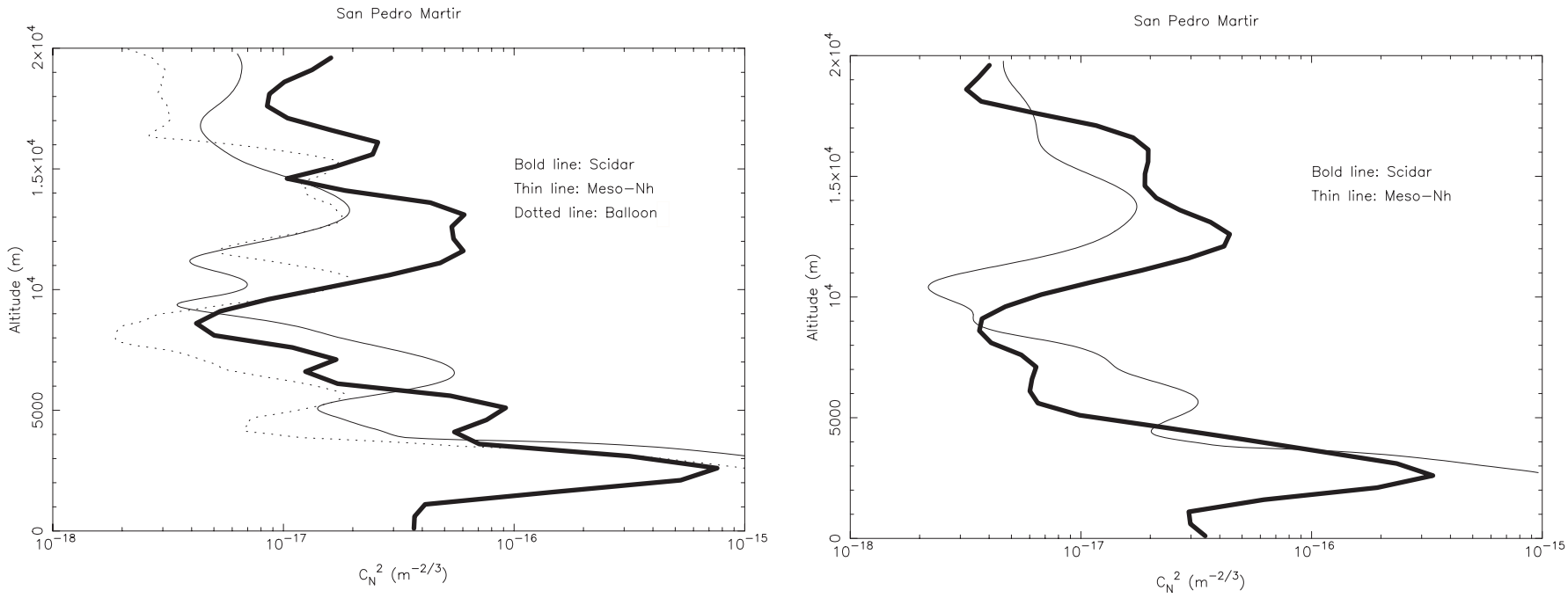

Fig. 4. On the left: $C_{\mathrm{N}}^{2}$ vertical profiles measured by the GS (bold line), the balloon (dotted line) and the $C_{\mathrm{N}}^{2}$ simulated by Meso-Nh (thin line) during the $A$ interval. On the right: $C_{\mathrm{N}}^{2}$ vertical profiles measured by the GS (bold line) and simulated by Meso-Nh (thin line) during the interval $B$.

Table 1. Summary of the seeing values obtained integrating the measured and simulated $C_{\mathrm{N}}^{2}$ in the interval $A$ and $B$ (see Fig. 4). $\varepsilon_{[0-10]}$ is the integration in the first $10 \mathrm{~km}, \varepsilon_{[10-20]}$ is the value obtained integrating between 10 and $20 \mathrm{~km}$. $\varepsilon_{\mathrm{TOT}}$ is the seeing integrated over the whole atmosphere. The mast values are related to the interval [2-16] m. The (DIMM-mast) value is calculated linearly with respect to the $C_{\mathrm{N}}^{2}$. The last four columns shows the difference of the seeing calculated between the Meso-Nh and the GS (MG), the Meso-Nh and the DIMM (MD), the GS and the DIMM (GD) and the GS and the balloons.

\begin{tabular}{|c|c|c|c|c|c|c|c|c|c|c|}
\hline Time (UT) & Parameter & $\begin{array}{l}\text { GS } \\
\left({ }^{\prime \prime}\right)\end{array}$ & $\begin{array}{c}\text { Meso-Nh } \\
\left({ }^{\prime \prime}\right)\end{array}$ & $\begin{array}{c}\text { DIMM } \\
\left({ }^{\prime \prime}\right)\end{array}$ & $\begin{array}{c}\text { mast }[2-16] \mathrm{m} \\
\left({ }^{\prime \prime}\right)\end{array}$ & $\begin{array}{c}\text { DIMM-mast } \\
\left({ }^{\prime \prime}\right)\end{array}$ & $\begin{array}{c}\Delta \varepsilon_{\mathrm{MG}} \\
\left({ }^{\prime \prime}\right)\end{array}$ & $\begin{array}{c}\Delta \varepsilon_{\mathrm{MD}} \\
\left({ }^{\prime \prime}\right)\end{array}$ & $\begin{array}{c}\Delta \varepsilon_{\mathrm{GD}} \\
\left({ }^{\prime \prime}\right)\end{array}$ & $\begin{array}{c}\Delta \varepsilon_{\mathrm{GB}} \\
\left({ }^{\prime \prime}\right)\end{array}$ \\
\hline$[7-8]$ & $\varepsilon_{[0-10]}$ & 1.32 & 1.35 & & & & 0.03 & & & - \\
\hline$"$ & $\varepsilon_{[10-20]}$ & 0.58 & 0.28 & & & & 0.30 & & & 0.28 \\
\hline$"$ & $\varepsilon_{\mathrm{TOT}}$ & 1.51 & 1.41 & 1.7 & 0.28 & 1.60 & 0.10 & 0.29 & 0.20 & - \\
\hline [11-12] & $\varepsilon_{[0-10]}$ & 0.86 & 0.88 & & & & 0.02 & & & - \\
\hline$"$ & $\varepsilon_{[10-20]}$ & 0.45 & 0.28 & & & & 0.17 & & & - \\
\hline$"$ & $\varepsilon_{\mathrm{TOT}}$ & 1.02 & 0.96 & 0.91 & 0.19 & 0.87 & 0.06 & 0.05 & 0.11 & - \\
\hline
\end{tabular}
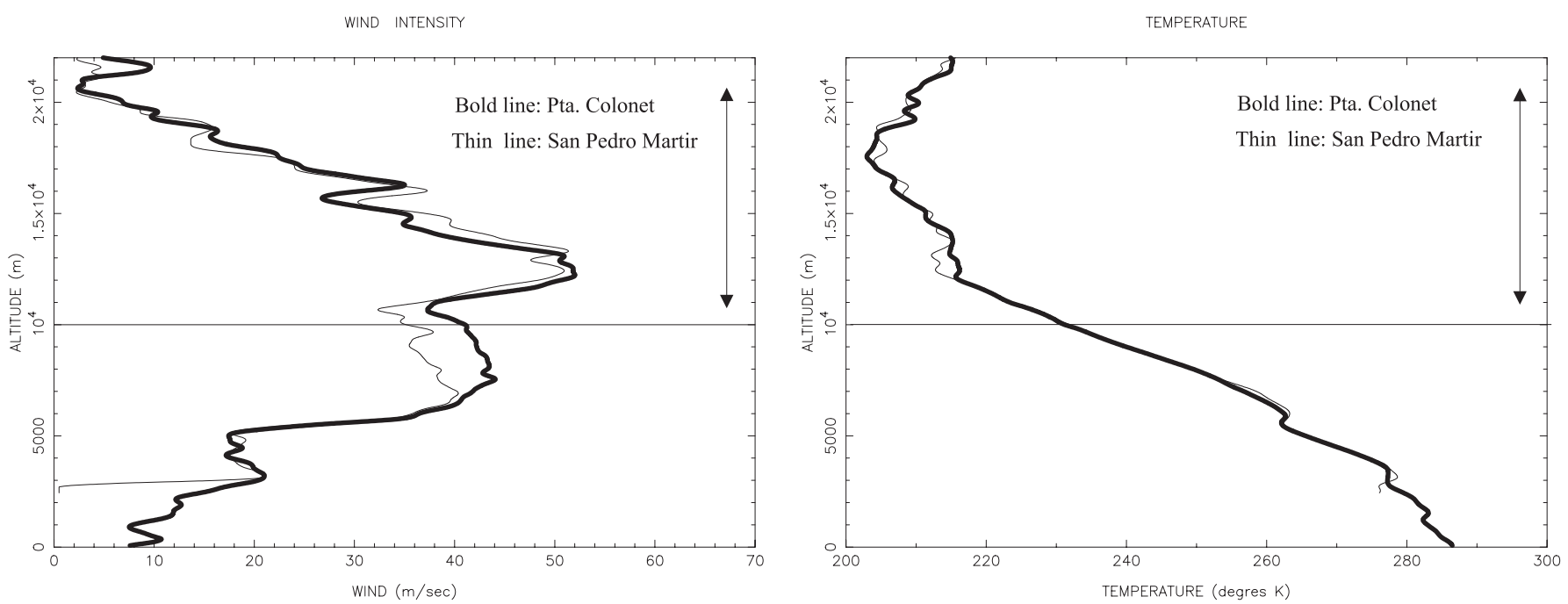

Fig. 5. On the left: vertical wind velocity profile measured by the balloon launched from the coast (Pta. Colonet, bold line) and from San Pedro Mártir (thin line). On the right: vertical profile of the absolute temperature. 

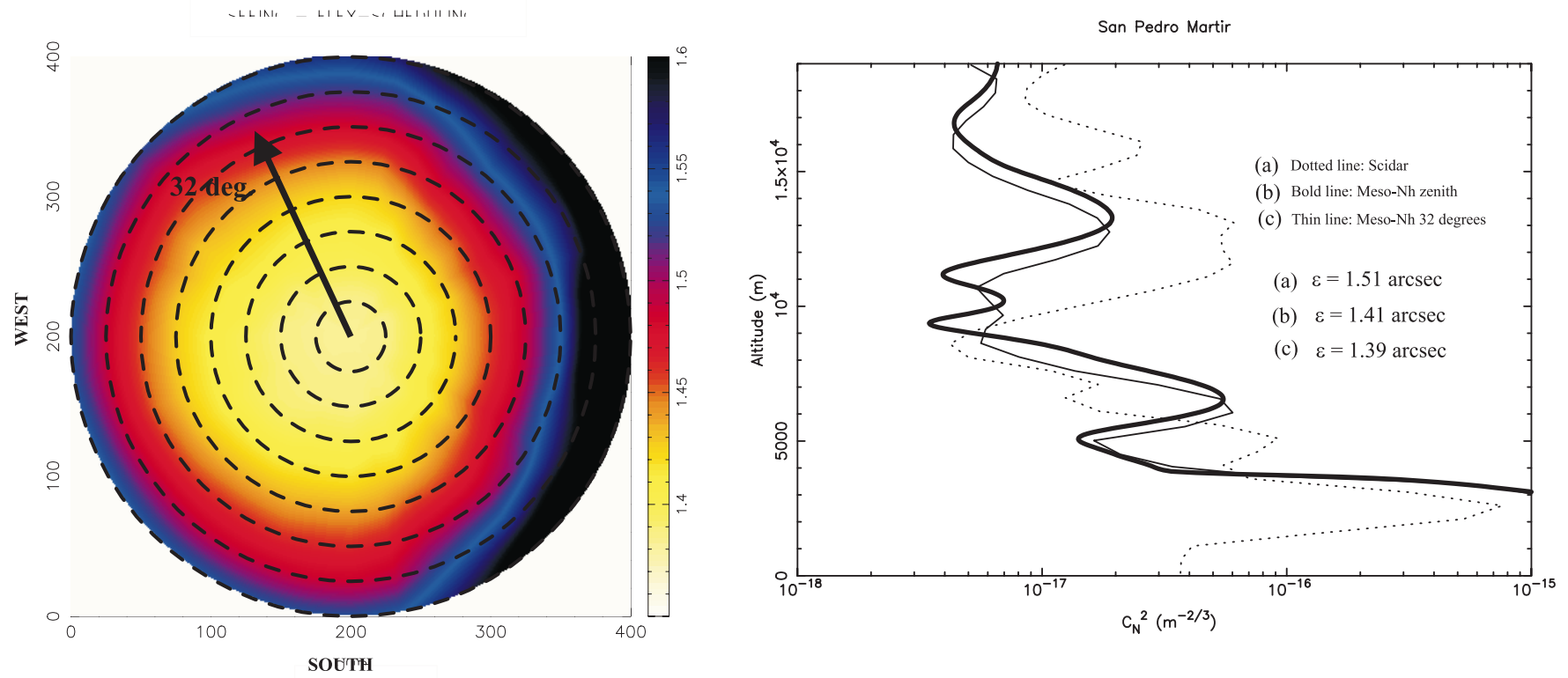

Fig. 6. On the left: polar map of the seeing simulated above the site during the interval $A$. The centre is the seeing integrated along the zenith. Each circle (increment of 5 degrees) is the seeing calculated at the same angle $\theta$ respect to the zenith. On the top is the geographic North, on the left, the geographic West. The color table shows a large seeing with the color black and a low seeing with the color yellow. The arrow indicates the direction and the amplitude of the angle $\theta$ of $\zeta$ UMa as shown in Fig. 3. On the right are shown the $C_{\mathrm{N}}^{2}$ profile measured by the GS (dotted line), the $C_{\mathrm{N}}^{2}$ profile simulated by Meso-Nh along the zenith (bold line) and along the same azimuth and elevation as shown in this same figure on the left (thin line).

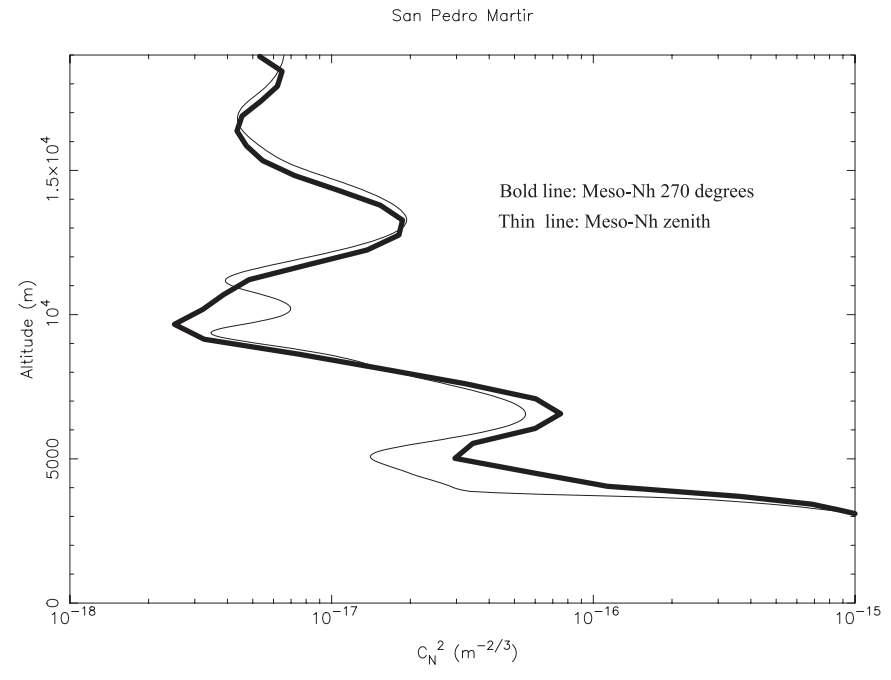

Fig. 7. $C_{\mathrm{N}}^{2}$ profiles simulated along the zenith (thin line) and at (270 degrees, $\theta=40$ degrees) (bold line) during the 16/17 night.

telescope $(2800 \mathrm{~m})$. Thus, it is not possible to calculate a reliable $\varepsilon_{[0-10]}$. We find that the seeing estimated by the GS and Meso-Nh is well correlated with the (DIMM - mast) measurements, that are equivalent to the integration of the $C_{\mathrm{N}}^{2}$ between the telescope dome and the top of the atmosphere. The shape of the GS and balloon $C_{\mathrm{N}}^{2}$ profiles is well correlated with that provided by Meso-Nh and the intensity of the turbulence in each layer is comparable. In the interval $A$, above about $10 \mathrm{~km}$, the simulated $C_{\mathrm{N}}^{2}$ profile seems to be more similar to the balloon than to the GS profile. After a detailed analyses of all the measurements it seems that the GS might have overestimated the $C_{\mathrm{N}}^{2}$ at this altitude. We note, in favor of this hypothesis, that the vertical profiles of the absolute temperature and wind velocity of the two balloons launched from the site and from the coast are exceptionally well correlated above $10 \mathrm{~km}$ (Fig. 5). The $C_{\mathrm{N}}^{2}$ depends directly on the gradient of the temperature and the wind velocity (Coulman et al. 1995) so we deduce that the meteorological parameters and probably the optical turbulence during this night were relatively uniform above $10 \mathrm{~km}$. This is consistent with a good correlation between the simulated $C_{\mathrm{N}}^{2}$ and that measured by the balloon. From this set of analyses we are confident in the Meso-NH simulations. Moreover, in the last four columns of Table 1, we note that the differences estimated for the seeing between the model and the different instruments are comparable. This assure a sufficient level of reliability of the model. Interestingly, we note that Meso-Nh can discriminate two time intervals of the same night characterized by strongly different turbulent conditions.

A question remains without answer: could the suspected GS overestimation at about $12 / 13 \mathrm{~km}$ in reality be due to the fact that the observations line of sight is strongly different from the zenith direction?

To investigate this we simulate, in the interval $A$, the $C_{\mathrm{N}}^{2}$ along the same line of sight as the observed one. Figure 6 (on the left) shows the polar seeing map (PSM). The centre corresponds to the integration of the $C_{\mathrm{N}}^{2}$ with 

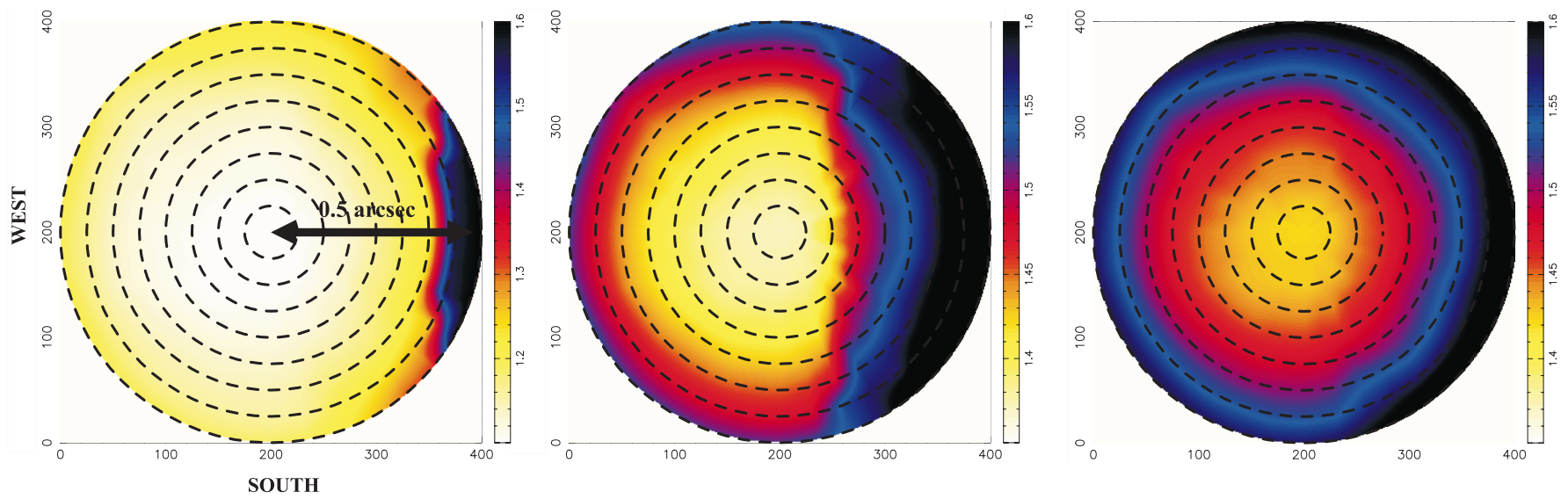

Fig. 8. Polar maps of seeing obtained at different instants (starting from the left: $t_{1}=950 \mathrm{~s}, t_{2}=2150 \mathrm{~s}$ and $t_{3}=2163 \mathrm{~s}$ ).

respect to the zenith. Each circle represents the seeing integrated at the same angle $\theta$ from the zenith. The increment in $\theta$ (that is the radius of the circles) is of 5 degrees. On the top is the geographic North, on the left, the West. The arrow indicates the direction and the amplitude of the angle $\theta$ of $\zeta$ UMa as shown in Fig. 3. We note that the air mass factor $\left((\cos \theta)^{-5 / 3}\right)$ is considered in the calculation of the seeing in the polar maps. In this way we can appreciate the total fluctuations provided both by the air mass factor and by the turbulent horizontal distribution. Figure 6 (on the right) shows the $C_{\mathrm{N}}^{2}$ profile measured by the GS (dotted line - (a)), the $C_{\mathrm{N}}^{2}$ profile simulated by Meso-Nh along the zenith (bold line - (b)) and along the same azimuth and elevation as shown by the arrow in this same figure on the left (thin line - (c)). We observe that the shape of the two simulated $C_{\mathrm{N}}^{2}$ profiles is similar and that the quantitative estimation of the two $C_{\mathrm{N}}^{2}$ profiles is comparable. This suggests that on this night, and along the direction fixed by the $\zeta$ UMa position, the horizontal size of the turbulent layers is large and the horizontal structure is fundamentally uniform. This result confirms the hypothesis expressed above about the overestimation of the GS in the higher part of the atmosphere. We therefore think that the fluctuations of the seeing observed in the PSM (Fig. 6 on the left) are due to a different turbulent distribution in the lower part of the atmosphere. By comparing the seeing values in the East (270 degrees) and West (90 degrees) direction (Fig. 6 on the left) we find differences of about $0.10^{\prime \prime}$ at about $\theta=40$ degrees. These fluctuations cannot be due to an air mass factor. Unfortunately, the strongest difference in the seeing on the PSM occurs along directions that are different from those of the observations. Figure 7 shows (bold-line) a simulated $C_{\mathrm{N}}^{2}$ in the East direction (270 degrees) and (thin-line) a simulated $C_{\mathrm{N}}^{2}$ along the zenith. The turbulence in the first $10 \mathrm{~km}$ seems effectively stronger in the East direction than respect to the zenith. Because of a lack of measurements at the 270 degrees direction we cannot confirm this result, but the simulations indicate that the horizontal size of turbulent layer in the first $10 \mathrm{~km}$ is finite and that the turbulent structure is not uniform, at least during this night.
For the adaptive optics application it is fundamental to know what is the order of magnitude of the horizontal spatial modulations of the PSMs. Figure 6 (on the left) shows the result of an average calculated over 3 simulation hours. This is the correct procedure to compare measurements with simulations but, in this way, we lose the maximum and minimum of the fluctuations. Figure 8 shows PSMs calculated at different instants during the 3 hours. Starting from the left we have $t_{1}=950 \mathrm{~s}, t_{2}=2150 \mathrm{~s}$ and $t_{3}=2163 \mathrm{~s}$. It can be seen that the seeing modulation is different in each PSM but a general common feature is observed in all of the maps: a strong turbulence production is seen on the East side. The sequence of these maps reproduces in a better way the real temporal evolution of the turbulence. We note that the amplitude of the seeing modulation can attain values of $0.50^{\prime \prime}$ over 40 degrees (Fig. 8, PSM on the left). This is a large modulation that cannot be due only to the air mass factor. This means that, in the real temporal evolution of the turbulence, large fluctuations of the seeing would be probably observed. This supports the thesis of a finite horizontal size of the turbulent layers, at least in the first $10 \mathrm{~km}$.

\subsection{Experimental validation}

An alternative possibility for inferring information on the horizontal size of the turbulent layers is the analysis of measurements provided by a GS. These measurements were expressly planned for such a study: we chose a set of different binary stars in order to scan different regions of the sky, and we averaged the measurements over time intervals that are sufficiently long to have a reliable statistical estimation of the turbulence in a fixed direction and sufficiently short to consider that the measurements are almost instantaneous.

The measurements of the night 21-22 May are analyzed. We analyze the [3.16/5] UT and the [7/9.16] UT intervals. Figure 9 (on the left) shows the temporal evolution of the $C_{\mathrm{N}}^{2}$ measured by the GS during the [7/9.16] UT interval. Three different binary stars were observed: $\zeta \mathrm{UMa}$, $\zeta$ Cbr $\left(\alpha_{2000}=15^{\mathrm{h}} 39^{\prime} 24^{\prime \prime}, \delta_{2000}=36^{\circ} 38^{\prime} 3^{\prime \prime}\right)$ and 95 Her. Figure 10 (on the right) shows the position of the three 

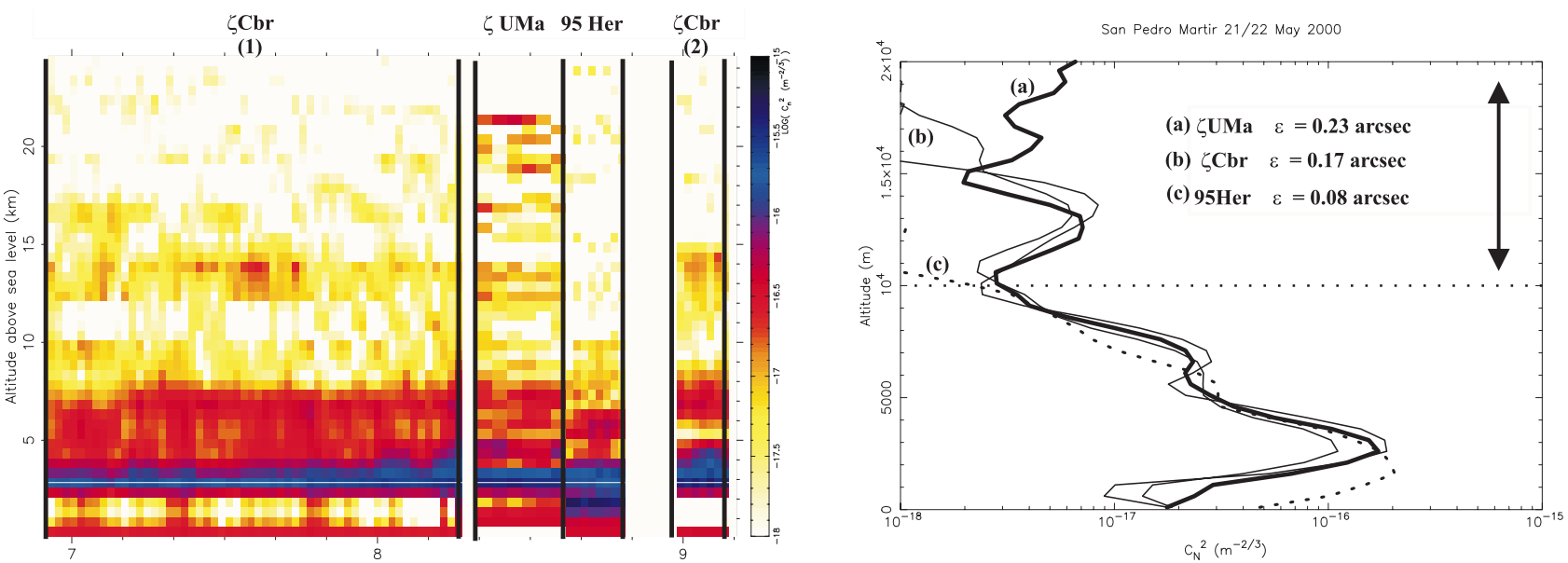

Fig. 9. On the left: temporal evolution of the $C_{\mathrm{N}}^{2}$ profiles measured by the GS during the night 21/22 May 2000 in the [7 - 9.16] UT time interval. The different binary stars observed by the GS are marked on the top of the figure. On the right: vertical $C_{\mathrm{N}}^{2}$ profiles corresponding to the 4 time intervals selected in the same figure on the left. The values of the seeing are calculated in the interval $[10-20] \mathrm{km}$.
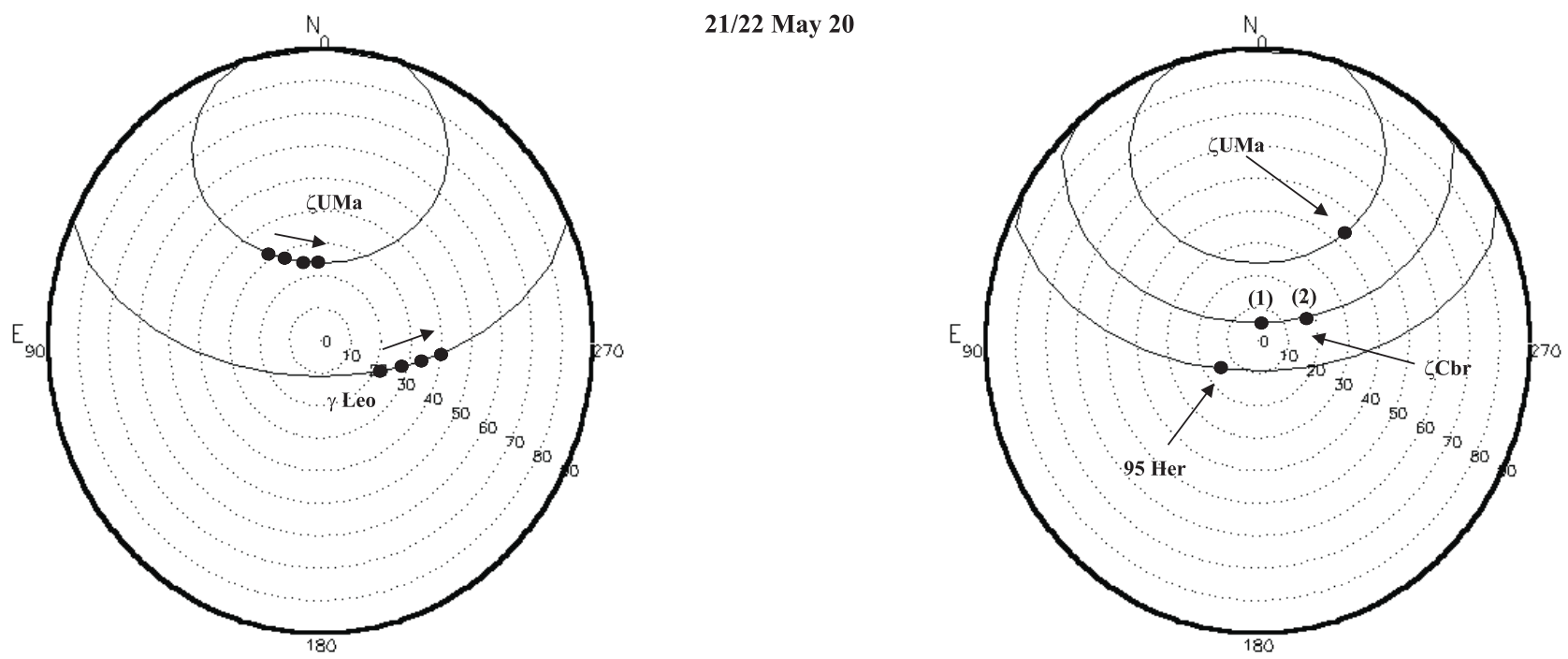

Fig. 10. Polar sky maps representing the path in the sky of the binary stars observed during the night $21 / 22$ (see Fig. 3 ). On the right: $\zeta \mathrm{Cbr}, \zeta \mathrm{UMa}$ and $95 \mathrm{Her}$ are observed during the interval [7/9.16] UT (1) and (2) refer to different temporal intervals (see Fig. 9). Each black dot marks the mean of the corresponding temporal interval. On the left: $\zeta$ UMa and $\gamma$ Leo were observed during the interval [3.16/5] UT The black dots mark the sequence of the intervals shown in Fig. 11.

stars in the sky at the instants of the GS observations. Figure 9 (on the right) shows the $C_{\mathrm{N}}^{2}$ vertical profiles averaged over each time interval selected on the left of the same figure. We note that, in the direction of $95 \mathrm{Her}$, the turbulence has a vertical distribution which is strongly different from the other cases. Looking at 95Her, the GS measures a stronger turbulence near the ground and the seeing integrated above $10 \mathrm{~km}$ is smaller than that calculated in the direction of $\zeta \mathrm{UMa}$ and $\zeta \mathrm{Cbr}$. The turbulent layer detected in the cases (a) and (b) in Fig. 9 above $10 \mathrm{~km}$ is completely absent in the case (c). Above $15 \mathrm{~km}$ a difference in turbulence profile also is observed between the $\zeta \mathrm{UMa}$ and $\zeta \mathrm{Cbr}$ directions. Above $10 \mathrm{~km}$ we measure a $\Delta \varepsilon_{\max }$ between the cases (a), (b) and (c) of $0.15^{\prime \prime}$. Although this difference is not quantitatively high, some parameters such as the isoplanatic angle would be affected in a strong way by the measured $\Delta \varepsilon_{\max }$.

Figure 11 is equivalent to Fig. 9 but the observed binary stars are $\gamma$ Leo $\left(\alpha_{2000}=10^{\mathrm{h}} 20^{\prime} 0^{\prime \prime}, \delta_{2000}=19^{\circ} 50^{\prime} 21^{\prime \prime}\right)$ and $\zeta \mathrm{UMa}$. On the right hand side are shown two vertical $C_{\mathrm{N}}^{2}$ profiles. The bold profile is obtained averaging all the measured $C_{\mathrm{N}}^{2}$ profiles provided by $\zeta \mathrm{UMa}$ (see left hand side of Fig. 11). The thin profile is obtained averaging all the measured $C_{\mathrm{N}}^{2}$ profiles provided by $\gamma$ Leo. We observe that, in the first $5 \mathrm{~km}$ and above $15 \mathrm{~km}$ the shape of the $C_{\mathrm{N}}^{2}$ profile is clearly different in the two cases. We estimate a $\Delta \varepsilon_{\max }=0.26^{\prime \prime}$ in $[0-10] \mathrm{km}$ and a $\Delta \varepsilon_{\max }=0.12^{\prime \prime}$ in [10-20] km.

Table 2 shows the seeing values calculated in the $[0-10] \mathrm{km}$ and $[10-20] \mathrm{km}$ regions related to all the intervals shown in Fig. 11. We observe a systematic difference 

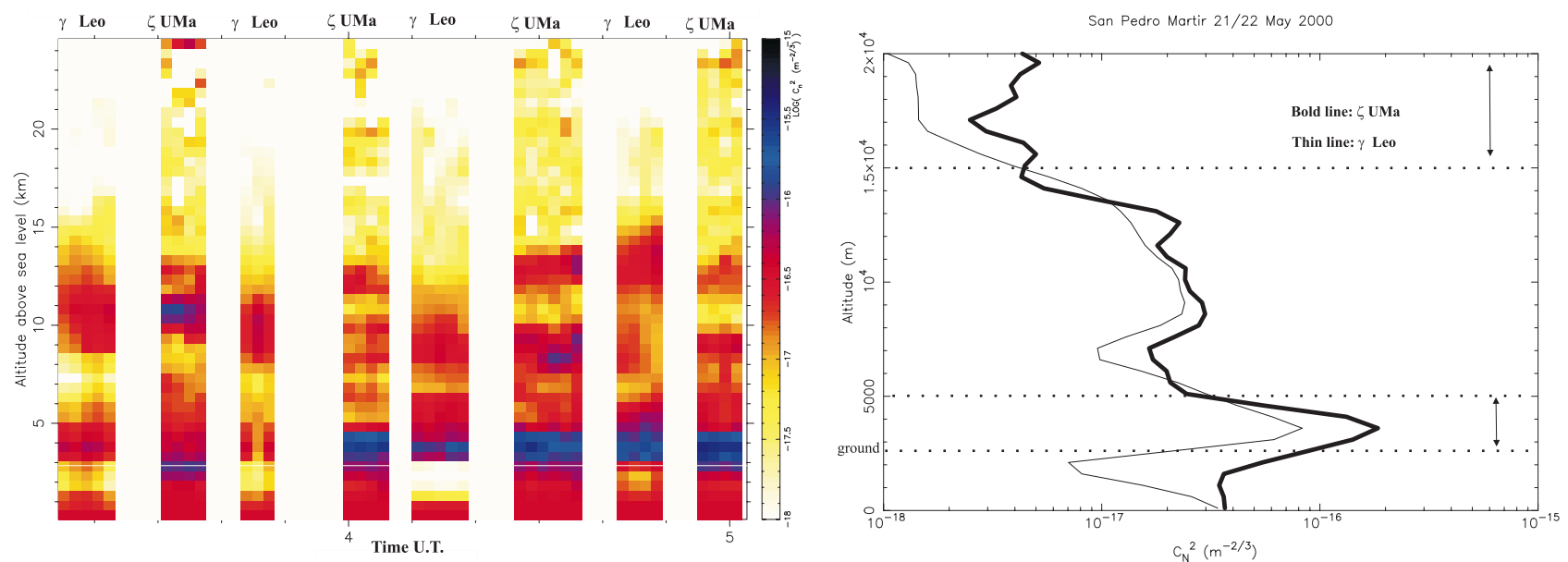

Fig. 11. On the left: as in Fig. 6 but in the [3.16/5] time interval. On the right: vertical $C_{\mathrm{N}}^{2}$ profiles obtained averaging the measured for the $\gamma$ Leo and $\zeta$ UMa directions over the all 4 intervals.

Table 2. Summary of the seeing values calculated in [0-10] $\mathrm{km}$ and [10-20] $\mathrm{km}$ for each interval shown in Fig. 11.

\begin{tabular}{ccccccccc}
\hline \hline Parameter $\left({ }^{\prime \prime}\right)$ & $\gamma$ Leo & $\zeta$ UMa & $\gamma$ Leo & $\zeta$ UMa & $\gamma$ Leo & $\zeta$ UMa & $\gamma$ Leo & $\zeta$ UMa \\
\hline \hline$\varepsilon_{[0-10]}$ & 0.38 & 0.58 & 0.38 & 0.76 & 0.58 & 0.84 & 0.71 & 0.91 \\
$\varepsilon_{[10-20]}$ & 0.30 & 0.44 & 0.24 & 0.27 & 0.20 & 0.31 & 0.35 & 0.26 \\
$\varepsilon_{\text {TOT }}$ & 0.52 & 0.78 & 0.48 & 0.84 & 0.63 & 0.93 & 0.83 & 0.97 \\
\hline \hline
\end{tabular}

in seeing estimations with respect to the $\gamma$ Leo and $\zeta \mathrm{UMa}$ directions in the higher and in the lower part of the atmosphere. This can be considered as a further proof of the finite horizontal size of the turbulent layers. Contrary to the case of the 16/17 night, it seems that this is detected over the whole troposphere $[0-20] \mathrm{km}$. We note that the differences in vertical $C_{\mathrm{N}}^{2}$ structure are observed, at least in the $21 / 22$ night, near the ground and in the higher part of the atmosphere. Beside this, it has been shown that the statistical noise, that intrinsically affects the GS measurements, increases near the ground and in the high part of the atmosphere (Vernin \& Azouit 1983; Tokovinin 1997; Prieur et al. 2001). Therefore we performed a deeper study to confirm that the seeing variations estimated in our study are effectively due to a real different distribution of the turbulence and not to some noise. We refer the reader to the Prieur et al. paper (2001) for more details related to the following discussion. Here we note only that the statistical noise is given by the uncertainty per frame of the optical turbulence factor $\Delta \widetilde{J}_{j}$ (Eq. (14) of the paper by Prieur et al. 2001):

$\Delta \widetilde{J}_{j}=\frac{5.23 \cdot 10^{-2} \lambda^{5 / 3} d_{j}{ }^{-5 / 6} d_{0}{ }^{1 / 2}\left(C_{2}(0)+N_{\mathrm{ph}}\right)}{B \cdot D \cdot \sqrt{f(x)}}$

where $\lambda$ is the wavelength, $C_{2}(0)$ the normalized covariance function of the irradiance evaluated in the origin, $d_{j}$ the distance of the turbulent layers from the plane where the scintillation is measured, $d_{0}$ the distance from the analysis plane to the layer that contributes the most to the scintillation, $N_{\mathrm{ph}}$ the mean number of photons per coherence area, $B$ is a function of the difference in magnitude $\Delta m$ of the binary stars, $D$ the pupil size, $x=\beta d_{j} / D, \beta$ the angular separations of the binary stars and $f(x)=\arccos (x)-x\left(1-x^{2}\right)^{1 / 2}$. For $N$ independent frames, $\Delta \widetilde{J}_{j}$ is multiplied by $N^{-1 / 2}$. Figure 12 shows, on the left, the function $\Delta \widetilde{J}_{j}$ calculated for $\zeta \mathrm{UMa}, \zeta \mathrm{Cbr}$ and $95 \mathrm{Her}$ of Fig. 9, on the right, the function $\Delta \widetilde{J}_{j}$ calculated for $\zeta$ UMa and $\gamma$ Leo of Fig. 11. We used a pupil size of $2.1 \mathrm{~m}$ corresponding to the telescope of San Pedro Mártir and $N=2000$ for all the cases. We underline that, during the observations, we used $N=2000$. Table 3 shows the values of $\Delta m,\left(C_{2}(0)+1 / N_{\mathrm{ph}}\right)$ and $d_{0}$ used for the different binary stars to obtained the Fig. 12. On the abscissas is shown the distance from the plane on which the scintillation is measured. The origin corresponds to $3000 \mathrm{~m}$ under the ground. The pupil of the telescope is marked with a vertical line at $3000 \mathrm{~m}$ from the origin in the Fig. 12. On the ordinates is shown the statistical noise. We note that, in the paper by Prieur et al. (2001), the origin corresponds to the telescope pupil, in our case to $3000 \mathrm{~m}$ under the ground. This is due to the fact that those measurements were made with a Classical Scidar whereas ours are made with a Generalized Scidar. We observe that, for all the binary stars observed, the statistical error is smaller than $10^{-15} \mathrm{~m}^{1 / 3}$ over the whole atmosphere. Knowing that $10^{-15} \mathrm{~m}^{1 / 3}$ corresponds to about $0.02^{\prime \prime}$ we conclude that the differences in seeing estimations found in Figs. 9 and 11 are not due to a statistical error and they can be considered as real.

\section{Implications for adaptive optics techniques}

We report here only some of the contexts in which such studies could be useful, basing our comments on the actual 

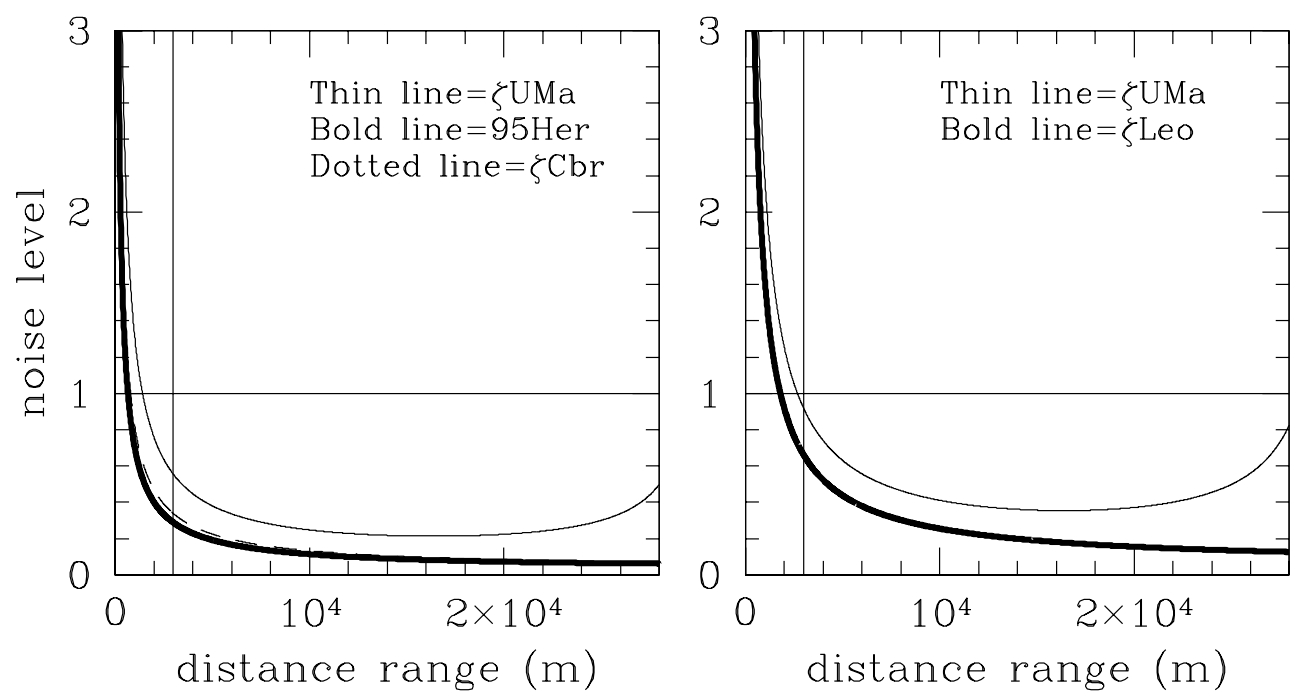

Fig. 12. Uncertainty of the optical turbulence factor $\Delta \widetilde{J}_{j}$ (in $10^{-15} \mathrm{~m}^{1 / 3}$ ) calculated (on the left) for the binary stars of Fig. 9 , (on the right) for the binary stars of Fig. 11. The vertical line marks the telescope pupil position.

Table 3. Summary of the values of $\Delta m,\left(C_{2}(0)+1 / N_{\mathrm{ph}}\right)$ and $d_{0}$ used to obtain the curves of Fig. 12. See the text.

\begin{tabular}{cccc}
\hline \hline Binary Star & $\Delta m$ & $\left(C_{2}(0)+1 / N_{\mathrm{ph}}\right)$ & $d_{0}(\mathrm{~m})$ \\
\hline \hline$\gamma$ Leo & 1.06 & 0.11 & 4000 \\
$\zeta \mathrm{UMa}$ & 1.62 & 0.12 & 4000 \\
\hline \hline$\zeta \mathrm{Cbr}$ & 0.96 & 0.07 & 3000 \\
$\zeta \mathrm{UMa}$ & 1.62 & 0.08 & 3000 \\
$95 \mathrm{Her}$ & 0.38 & 0.07 & 3000 \\
\hline \hline
\end{tabular}

scientific debates and on the open problems related to the new generation of adaptive optics techniques such as the MCAO and the 3D turbulence tomography.

A. It was observed (Le Louarn et al. 2000; Hubin et al. 2000) that the spatio-temporal stability of the thin turbulence layers in the atmosphere is extremely important for an optimized application of the Multi-Conjugated Adaptive Optics (MCAO). Moreover, we infer from a previous study (Ragazzoni 1999a) that the most critical stability is that of the highest layers. This is true in general for all the classical Natural Guide Stars (NGS)-based adaptive optics systems and in particular for the NGSturbulence tomography in which the highest turbulent layers affect in a stronger way the maximum corrected field of view.

It is reasonable to think that the spatio-temporal fluctuations of the turbulence at the high altitudes are smaller than those at low altitudes because the orographic effects principally affect the lower part of the atmosphere. Bursts of thin turbulent layers at high altitudes are less frequent and are generally produced by synoptic phenomena (i.e., by large scale perturbations). For the same reason we can suppose that the typical horizontal size of the turbulent layers is larger in the higher part of the atmosphere than in the lower one.

Although these statements are in general true, the preliminary results presented in this paper seem to indicate that the modulations of the spatial distribution of the turbulence can be large for angular separations of about 40 degrees and that this is observed not only in the first kilometers above the ground but on the whole atmosphere $(20 \mathrm{~km})$. We therefore deduce that the typical horizontal size of the turbulent layer can be as important as the temporal stability of the layers.

B. The turbulence tomography (Tallon \& Foy 1990; Ragazzoni et al. 2000a) is one of the most promising techniques for the sensing and correction of the perturbed wavefront for the new class of large size telescopes (Ragazzoni 1999a; Hubin et al. 2000). It was proved (Ragazzoni et al. 1999b, Ragazzoni et al. 2000a) that the wavefront perturbations at an altitude $h$ can be described by a modal tomographic matrix $M$ that depends on the altitude and the relative strength of the perturbed layers in the whole atmosphere. This means that $M=M\left(C_{\mathrm{N}}^{2}\right)$. As remarked by the authors, the typical lifetime $\tau_{M}$ of the matrix $M$ and the time intervals to be used for its best estimation are a new problem that has to be solved for a real application of this technique. Our study would help to answer the following question: is it enough to give a characterization of the $C_{\mathrm{N}}^{2}(z)$ or do we need a more detailed characterization of $C_{\mathrm{N}}^{2}(x, y, z)$ ? In the latter case we should probably define a $\tau_{M}$ depending on the direction of the line of sight. Just as an example, on the Roque de Los Muchachos Observatory (Canary Islands), it is possible that the $\tau_{M}$ with respect to the southern direction is smaller than with respect to a northern direction. Because of the presence of the Caldera, the turbulence near the ground is particularly strong in the southern direction from the Observatory. 
C. If the results presented in this paper are confirmed, we would conclude that the optical turbulence vertical profilers (OTVPs) should have an extended sky coverage to correctly support the adaptive optics techniques. For this reason, a profiler that works with single stars would probably be more useful than a Generalized Scidar. As an example, it is enough to remember that, during the San Pedro Mártir site testing campaign on May 2000, only the northern side of the sky sphere was scanned by the Generalized Scidar (see Figs. 3 and 10) because of a lack of appropriate binaries in other directions.

D. Recently, Ragazzoni (1999a) investigated the interesting possibility, in the context of the turbulence tomography, of avoiding the employment of LGS for the correction of the wavefront in the case of large size telescopes. The sky coverage of the NGS would be enough under particular conditions. Using such a technique, the maximum size $\theta$ of the tomographic corrected field of view is (Hubin et al. 2000; Le Louarn et al. 2000):

$\theta_{D, h_{\max }}=\frac{D}{h_{\max }}\left(1-\frac{h_{\max }}{H}\right)$

where $D$ is the diameter of the telescope and $h_{\max }$ is the height of the highest turbulent layer. $H$ is the altitude of the reference star $(H=90 \mathrm{~km}$ in the LGS case and $H$ is infinity in the case of NGS). The field of view is strongly dependent on the altitude of the highest turbulent layer. In Table 4 are shown the values of the corrected field of view for different values of $h_{\max }$. In the first two columns the values of $\theta$ obtained are shown for a telescope of $100 \mathrm{~m}$ and $8 \mathrm{~m}$ in the NGS case. In the third and fourth column the values obtained for the LGS case are shown. In the last line we report, for each case, the gain which is defined as:

$G=\frac{\theta_{D, 15 \mathrm{~km}}}{\theta_{D, 20 \mathrm{~km}}}$

We calculate that, for a $100 \mathrm{~m}$ pupil size, $\theta$ can gain (or lose) a factor equivalent to $77 \%$ (in the NGS case) and $72 \%$ (in the LGS case) if the $h_{\max }$ changes from 20 to $15 \mathrm{~km}$ (or from 15 to $20 \mathrm{~km}$ ). This means that the maximum field of view could have different sizes (quantitatively not negligible) for different regions in the sky. We note that, as pointed out by Le Louarn et al. (2000), the value of $\theta_{D, h_{\max }}$ and $G$ could change if we consider a different definition of the $\mathrm{FOV} \theta$.

We finally note that, in a more recent papers (Rigault et al. 2000), the authors observed that a field of view of the order of those calculated in our paper should require a too large number of DMs to correct the perturbed wavefront. This is due to the fact that, the larger the FOV, the higher the number of the DMs necessary to keep the MCAO errors (generalized fitting and anisoplanetism errors) down. Besides this, we think that it is not desirable to limit ourself to such a technical obstacle. New techniques could be proposed in the future by passing the limitation imposed by the number of DMs. For example, alternative solutions such as the layer oriented MCAO (Ragazzoni et al. 2000b;
Table 4. Maximum field of view obtained with the 3D tomographic technique (Ragazzoni et al. 2000a) calculated for different pupil sizes and for different values of the altitude of the highest turbulent layer $h_{\max }$. The last line shows the gain calculated using Eq. (2).

\begin{tabular}{ccccc}
\hline \hline$h_{\max }$ & $\begin{array}{c}\theta_{100 \mathrm{~m}}\left(^{\prime}\right) \\
\text { NGS }\end{array}$ & $\begin{array}{c}\theta_{8 \mathrm{~m}}\left(^{\prime \prime}\right) \\
\text { NGS }\end{array}$ & $\begin{array}{c}\theta_{100 \mathrm{~m}}\left(^{\prime}\right) \\
\text { LGS }\end{array}$ & $\begin{array}{c}\theta_{8 \mathrm{~m}}\left(^{\prime \prime}\right) \\
\text { LGS }\end{array}$ \\
\hline \hline 20 & 17 & 80 & 13 & 62 \\
18 & 18 & 89 & 14 & 71 \\
15 & 22 & 106 & 18 & 89 \\
\hline Gain & $77 \%$ & $75 \%$ & $72 \%$ & $70 \%$ \\
\hline \hline
\end{tabular}

Diolaiti et al. 2001) were recently proposed. The technique seems to be stable (Diolaiti et al. 2001) and hopeful but the sky coverage obtained with only NGSs has not yet been calculated in this case. Unfortunately, it seems that the problem of the large number of DMs necessary to correct a large FOV has still to be solved.

\section{Conclusions}

In this paper we try to validate, following different approaches, the thesis that the horizontal size of turbulent layers is finite, and we discuss the implications that this fact can have on adaptive optics techniques. $C_{\mathrm{N}}^{2}$ numerical simulations and measurements are used. To obtain a more reliable comparison between measurements and simulations we adapted the code in order to retrieve estimations of the $C_{\mathrm{N}}^{2}$ profiles simulated with respect to lines of sight different from the zenith and averaged over some hours. The conclusions are the following:

- We showed that the simulated $C_{\mathrm{N}}^{2}$ profiles are well correlated with the measured ones (GS and balloons) from a qualitative and a quantitative point of view. Unfortunately, the case studied (16/17 night) reveals a general uniformity of the stratification of the turbulent layers in the direction of the observed binary stars. Beside this, our simulations show that the typical horizontal size of the turbulent layers in the first $10 \mathrm{~km}$ is finite and can produce, along different directions, variations of the seeing estimations that, quantitatively, cannot be neglected. This last point cannot be confirmed by our measurements because there are regions in the atmosphere which were not scanned by the GS. On the other hand, as in a previous paper (Masciadri et al. 2000), the simulations seem to indicate a finite horizontal size of the optical turbulence.

- We quantified the intensity of the spatial fluctuations of the seeing maps. For the night of 16/17 May 2000 we find fluctuations of the order of $0.20^{\prime \prime}$ over a maximum angle of 40 degrees. In the simulated instantaneous seeing maps the spatial modulations can attain the order of $0.50^{\prime \prime}$.

- From a detailed comparison between simulations and a set of measurements provided by different instruments 
we show that the simulations agree well with the measurements. This proves the strength of the numerical tool. Obviously, the principal challenge for the model is to provide reliable estimations of the $C_{\mathrm{N}}^{2}$ without a systematic confirmation done with measurements.

- We showed that the $C_{\mathrm{N}}^{2}$ profiles measured by a GS at different angles from the zenith can have different shapes (that means different vertical distribution of the turbulence) and different total energies (a difference of $0.30^{\prime \prime}$ is estimated in the case of Fig. 11). After a study of the statistical noise related to our measurements, we conclude that the non-uniformity of the turbulent layers estimated in our study cannot be due to a statistical error and it has to be considered as real. It would be interesting to verify, in the future, if such a modulation is reproduced by the model.

- We show, for the first time, that Meso-Nh can reconstruct observed seeing values which differ by as much as $0.50^{\prime \prime}$ during the same night. In principle, a whole night can be covered by Meso-Nh simulations if the model is initialized with ECMWF products every 6 hours.

This is an encouraging result for a realistic employment of Meso-Nh in the optimal selection of scientific programs scheduled for observations in the new generation of large size telescopes.

- Further cross-correlations between measurements obtained with different instruments is suggested. The case of the night 16/17 presented in this paper shows clearly that there are, sometimes, inconsistencies between them.

Because the results obtained in this study are encouraging but not completely conclusive, we plan to carry out a deeper investigation. Two approaches are suggested: (1) to do 3D simulations of the atmosphere with the Meso-Nh model and to calculate the typical size of decorrelation of the turbulence with respect to the horizontal dimension. (2) to measure $C_{\mathrm{N}}^{2}$ profiles with respect to different directions using, at least, two Generalized Scidars. Moreover, to better study the horizontal size of turbulent layers, we planned to remove the air mass factor from the PSMs.

The good sensitivity of the Meso-Nh model in reconstructing $3 \mathrm{D}$ maps of $C_{\mathrm{N}}^{2}$ is now supported by many studies (Masciadri et al. 1999a; Masciadri et al. 1999b; Masciadri et al. 2001b). It is time to apply the numerical technique to a concrete problem in the context of adaptive optics techniques.

Acknowledgements. This work was supported by the TIM (Telescopio Infrarrojo Mexicano) Project, the CONACYT grant J32412E and the DGAPA grant IN118199. We thank P. Bougeault for kindly supplying CPU time for this study. We are grateful to all the people of Bougeault's team for their complete availability in supporting us in the handling of the
Meso-Nh model. We are indebted to J. Vernin's group from Nice University and M. Gizard (Centre National de Recherches Météorologiques - Meteo France, Toulouse - France) for participating in the observing campaign with their instruments (GS, balloons, mast). We aknowledge the anonymous referee for his useful commentaries.

\section{References}

Avila, R., Vernin, J., \& Masciadri, E. 1997, Appl. Opt., 36 7898

Avila, R., Vernin, J., \& Cuevas, S. 1998, PASP, 110, 1106

Beckers, J. M. 1988, ESO Workshop Proceedings - Very Large Telescopes and their instrumentation, 693

Coulman, C., Vernin, J., \& Fuchs, A. 1995, Appl. Opt., 34, 5461

Dalaudier, F., Sidi, C., Crochet, M., et al. 1994, J. Atm. Sci., 51, 237

Diolaiti, E., Ragazzoni, R., \& Tordi, M. 2001, A\&A, 372, 710

Fuchs, A., Tallon, M., \& Vernin, J. 1998, PASP, 110, 86

Hubin, N., et al. 2000, Adaptive Optical System Technology, SPIE Proc., 4007, 1100

Le Louarn, M., Hubin, N., Sarazin, M., et al. 2000, MNRAS, 317,535

Marks, R., Vernin, J., Azouit, M., et al. 1996, A\&AS, 118, 385

Marks, R., Vernin, J., Azouit, M., et al. 1999, A\&AS, 134, 161

Masciadri, E., Vernin, J., \& Bougeault, P. 1999a, A\&AS, 137, 185

Masciadri, E., Vernin, J., \& Bougeault, P. 1999b, A\&AS, 137, 203

Masciadri, E., et al. 2000, Interferometry in Optical Astronomy, SPIE Proc., 4006, 1136

Masciadri, E. 2001a, ASP Conf. Ser. - Astronomical Site Evaluation in the Visible and Radio Range, Marrakech 1317 November 2000, in press

Masciadri, E., Vernin, J., \& Bougeault, P. 2001b, A\&A, 365, 699

Prieur, J. L., Daigne, G., \& Avila, R. 2001, A\&A, 371, 366

Racine, R., \& Ellerbroek, B. L. 1995, Proc. SPIE, 2534, 248

Ragazzoni, R. 1999a, A\&A, 136, 205

Ragazzoni, R., Marchetti, E., \& Rigault, F. 1999b, A\&A, L53

Ragazzoni, R., Marchetti, E., \& Valente, P. 2000a, Nature, 403, 54

Ragazzoni, R., Farinato, J., \& Marchetti, E. 2000b, Proc. SPIE, 4007, 1076

Rigault, F., Ellerbroek, B. L., \& Flicker, R. 2000, Proc. SPIE, 4007, 1022

Sánchez, L., Avila, R., \& Masciadri, E. 2000, ASP Conf. Ser. - Astronomical Site Evaluation in the Visible and Radio Range, Marrakech 13-17 November 2000, in press

Tallon, M., \& Foy, R. 1990, A\&A, 235, 549

Tallon, M., Foy, R., \& Vernin, J. 1992, ESO Workshop Proceeding - Progress in Telescope and Instrumentation Technologies, 517

Tokovinin, A. A. 1997, VLT-TRE-UNI-17416-0003, ESO, Garching

Vernin, J., \& Azouit, M. 1983, J. Opt. (Paris), 14, 131 